\title{
Topological properties of regular generalized function algebras
}

\author{
H. Vernaeve* \\ 2000 Mathematics subject classification: $46 F 30$.

\begin{abstract}
We investigate density of various subalgebras of regular generalized functions in the special Colombeau algebra $\mathcal{G}(\Omega)$ of generalized functions.
\end{abstract}

\section{Introduction}

M. Oberguggenberger introduced the algebra $\mathcal{G}^{\infty}(\Omega)$ of regular generalized functions in order to develop a hypoelliptic regularity theory and hyperbolic propagation of singularities in the algebra $\mathcal{G}(\Omega)$ of Colombeau generalized functions [13, where it takes over the role of the subalgebra of $\mathcal{C}^{\infty}$-regular functions in the space $\mathcal{D}^{\prime}(\Omega)$ of distributions. It thus became the starting point of investigations of microlocal regularity in generalized function algebras (see [5, 7, 9, 10, 12, 16] and the references therein). More recently, various other subalgebras of regular generalized functions have been considered, from the point of view of generalized analytic functions [1], kernel theorems [3], propagation of singularities [14] and microlocal analysis [4]. We show that, in contrast with the situation of $\mathcal{C}^{\infty}(\Omega)$ as a subalgebra of $\mathcal{D}^{\prime}(\Omega)$ (and therefore maybe surprisingly), the subalgebra $\mathcal{G}^{\infty}(\Omega)$ and the subalgebras $\mathcal{G}_{\mathcal{L}_{a}}(\Omega)$ considered in [3, 4] are not dense in the algebra $\mathcal{G}(\Omega)$. On the other hand, the subalgebra of sublinear or S-analytic generalized functions is dense in $\mathcal{G}(\Omega)$.

\section{Notations}

Let $\Omega \subseteq \mathbb{R}^{d}$ be open. By $K \subset \subset \Omega$, we denote a compact subset of $\Omega$.

For $u \in \mathcal{C}^{\infty}(\Omega), K \subset \subset \Omega$ and $\alpha \in \mathbb{N}^{d}$, let $p_{\alpha, K}(u):=\sup _{x \in K}\left|\partial^{\alpha} u(x)\right|$. For $k \in \mathbb{N}$, let $p_{k, K}(u):=\max _{|\alpha|=k} p_{\alpha, K}(u)$.

The special algebra of Colombeau generalized functions (see e.g. [8]) is $\mathcal{G}(\Omega):=$ $\mathcal{E}_{M}(\Omega) / \mathcal{N}(\Omega)$, where

$$
\begin{aligned}
\mathcal{E}_{M}(\Omega)= & \left\{\left(u_{\varepsilon}\right)_{\varepsilon} \in \mathcal{C}^{\infty}(\Omega)^{(0,1)}:(\forall K \subset \subset \Omega)\left(\forall \alpha \in \mathbb{N}^{d}\right)(\exists N \in \mathbb{N})\right. \\
& \left.\left(p_{\alpha, K}\left(u_{\varepsilon}\right) \leq \varepsilon^{-N}, \text { for small } \varepsilon\right)\right\} \\
\mathcal{N}(\Omega)= & \left\{\left(u_{\varepsilon}\right)_{\varepsilon} \in \mathcal{C}^{\infty}(\Omega)^{(0,1)}:(\forall K \subset \subset \Omega)\left(\forall \alpha \in \mathbb{N}^{d}\right)(\forall m \in \mathbb{N})\right. \\
& \left.\left(p_{\alpha, K}\left(u_{\varepsilon}\right) \leq \varepsilon^{m}, \text { for small } \varepsilon\right)\right\} .
\end{aligned}
$$

*Dept. Of Mathematics, Ghent University. E-mail: hvernaev@cage.ugent.be 
By $\left[\left(u_{\varepsilon}\right)_{\varepsilon}\right]$, we denote the generalized function with representative $\left(u_{\varepsilon}\right)_{\varepsilon} \in \mathcal{E}_{M}(\Omega)$.

The subalgebra $\mathcal{G}_{c}(\Omega)$ of compactly supported generalized functions consists of those $u \in \mathcal{G}(\Omega)$ such that for some $K \subset \subset \Omega$, the restriction of $u$ to $\Omega \backslash K$ equals 0 (as an element of $\mathcal{G}(\Omega \backslash K)$ ).

For $K \subset \subset \Omega$, the algebra $\mathcal{G}^{\infty}(K)$ consists of those $u \in \mathcal{G}(\Omega)$ such that for one (and hence for each) representative $\left(u_{\varepsilon}\right)_{\varepsilon}$,

$$
(\exists N \in \mathbb{N})\left(\forall \alpha \in \mathbb{N}^{d}\right)\left(p_{\alpha, K}\left(u_{\varepsilon}\right) \leq \varepsilon^{-N}, \text { for small } \varepsilon\right) .
$$

For $\left(z_{\varepsilon}\right)_{\varepsilon} \in \mathbb{C}^{(0,1)}$, the valuation $\mathrm{v}\left(z_{\varepsilon}\right):=\sup \left\{b \in \mathbb{R}:\left|z_{\varepsilon}\right| \leq \varepsilon^{b}\right.$, for small $\left.\varepsilon\right\}$ and the so-called sharp norm $\left|z_{\varepsilon}\right|_{\mathrm{e}}:=e^{-\mathrm{v}\left(z_{\varepsilon}\right)}$. For $u \in \mathcal{G}(\Omega), P_{\alpha, K}(u):=\left|p_{\alpha, K}\left(u_{\varepsilon}\right)\right|_{\mathrm{e}}\left(\alpha \in \mathbb{N}^{d}\right)$ and $P_{k, K}(u):=\left|p_{k, K}\left(u_{\varepsilon}\right)\right|_{\mathrm{e}}(k \in \mathbb{N})$, independent of the representative $\left(u_{\varepsilon}\right)_{\varepsilon}$ of $u$. The ultra-pseudo-seminorms $P_{\alpha, K}\left(\alpha \in \mathbb{N}^{d}, K \subset \subset \Omega\right)$ determine a topology on $\mathcal{G}(\Omega)$ called sharp topology [2, 6, 16]. Then $u \in \mathcal{G}^{\infty}(K)$ iff $\sup _{k \in \mathbb{N}} P_{k, K}(u)<+\infty$. Further, the algebra $\mathcal{G}^{\infty}(\Omega):=\bigcap_{K \subset \subset \Omega} \mathcal{G}^{\infty}(K)[13]$.

For $K \subset \subset \Omega$, the algebra $\mathcal{G}_{\mathcal{L}_{a}}(K)$ of generalized functions of sublinear growth with slope smaller than $a>0(a \in \mathbb{R})$ on $K$ consists of those $u \in \mathcal{G}(\Omega)$ such that for one (and hence for each) representative $\left(u_{\varepsilon}\right)_{\varepsilon}$,

$$
\left(\exists a^{\prime}<a\right)(\exists b \in \mathbb{R})\left(\forall \alpha \in \mathbb{N}^{d}\right)\left(p_{\alpha, K}\left(u_{\varepsilon}\right) \leq \varepsilon^{-a^{\prime}|\alpha|-b}, \text { for small } \varepsilon\right)
$$

or, equivalently,

$$
\left(\exists a^{\prime}<a\right)(\exists c \in \mathbb{R})\left(P_{\alpha, K}(u) \leq c e^{a^{\prime}|\alpha|}, \forall \alpha \in \mathbb{N}^{d}\right),
$$

which can still be expressed concisely by $\lim \sup _{k \rightarrow \infty} \frac{\ln P_{k, K}(u)}{k}<a$. Since $P_{\alpha, K}(u v) \leq$ $\max _{\beta \leq \alpha}\left(P_{\beta, K}(u) P_{\alpha-\beta, K}(v)\right)$ by Leibniz's rule, $\mathcal{G}_{\mathcal{L}_{a}}(K)$ are subalgebras of $\mathcal{G}(\Omega)$.

For $a=0, \mathcal{G}_{\mathcal{L}_{0}}(K):=\bigcap_{a>0} \mathcal{G}_{\mathcal{L}_{a}}(K)$. Clearly, $\mathcal{G}^{\infty}(K) \subseteq \mathcal{G}_{\mathcal{L}_{0}}(K)$.

Again, the algebras $\mathcal{G}_{\mathcal{L}_{a}}(\Omega):=\bigcap_{K \subset \subset \Omega} \mathcal{G}_{\mathcal{L}_{a}}(K)(a \geq 0)$ [3, 4]. Clearly, $\mathcal{G}^{\infty}(\Omega) \subseteq \mathcal{G}_{\mathcal{L}_{0}}(\Omega)$. By definition, $u=\left[\left(u_{\varepsilon}\right)_{\varepsilon}\right] \in \mathcal{G}(\Omega)$ is sublinear [1, 15] iff for each $K \subset \subset \Omega$ and each $\left(x_{\varepsilon}\right)_{\varepsilon} \in K^{(0,1)}$, there exists $k \in \mathbb{R}$ and $\left(p_{n}\right)_{n \in \mathbb{N}} \in \mathbb{R}^{\mathbb{N}}$ such that $\lim _{n \rightarrow \infty} p_{n}+k n=\infty$ and for each $\alpha \in \mathbb{N}^{d},\left|\partial^{\alpha} u_{\varepsilon}\left(x_{\varepsilon}\right)\right| \leq \varepsilon^{p|\alpha|}$, for small $\varepsilon$. It can be shown [1, Thm. 5.7], [15, Thm. 10] that the algebra of sublinear generalized functions exactly contains those $u \in \mathcal{G}(\Omega)$ satisfying a natural condition of analyticity (called $S$-real analyticity in [15]). Sublinearity can still be characterized as follows by means of the algebras $\mathcal{G}_{\mathcal{L}_{a}}(K)$ :

Lemma 2.1. Let $u \in \mathcal{G}(\Omega)$. Then $u$ is sublinear iff for each $K \subset \subset \Omega$, there exists $a>0(a \in \mathbb{R})$ such that $u \in \mathcal{G}_{\mathcal{L}_{a}}(K)$.

Proof. $\Rightarrow$ : let $u$ be sublinear and suppose that there exists $K \subset \subset \Omega$ such that $u \notin \mathcal{G}_{\mathcal{L}_{a}}(K)$, for each $a>0$. Then we find $\alpha_{n} \in \mathbb{N}$ (for each $n \in \mathbb{N}$ ), $\varepsilon_{n, m} \in$ $(0,1 / m)$ (for each $n, m \in \mathbb{N}$ ) (by enumerating the countable family $\left(\varepsilon_{n, m}\right)_{n, m}$, we can successively choose the $\varepsilon_{n, m}$ such that they are all different) and $x_{\varepsilon_{n, m}} \in K$ such that $\left|\partial^{\alpha_{n}} u_{\varepsilon_{n, m}}\left(x_{\varepsilon_{n, m}}\right)\right|>\varepsilon_{n, m}^{-n\left|\alpha_{n}\right|-n}$, for each $n, m \in \mathbb{N}$. Let $x_{\varepsilon} \in K$ arbitrary if $\varepsilon \in(0,1) \backslash\left\{\varepsilon_{n, m}: n, m \in \mathbb{N}\right\}$. By assumption, there exist $k \in \mathbb{R},\left(p_{n}\right)_{n \in \mathbb{N}} \in \mathbb{R}^{\mathbb{N}}$ and $N \in \mathbb{N}$ such that for each $\alpha \in \mathbb{N}^{d}$ with $|\alpha| \geq N,\left|\partial^{\alpha} u_{\varepsilon}\left(x_{\varepsilon}\right)\right| \leq \varepsilon^{p_{|\alpha|}} \leq \varepsilon^{-k|\alpha|}$, for small $\varepsilon$. Since $u \in \mathcal{G}(\Omega)$, it follows that there exists $b \in \mathbb{R}$ such that for each $\alpha \in \mathbb{N}^{d}$, $\left|\partial^{\alpha} u_{\varepsilon}\left(x_{\varepsilon}\right)\right| \leq \varepsilon^{-k|\alpha|-b}$, for small $\varepsilon$. This contradicts the fact that for $n \in \mathbb{N}$ with $n \geq k$ and $n \geq b, \lim _{m} \varepsilon_{n, m}=0$ and $\left|\partial^{\alpha_{n}} u_{\varepsilon_{n, m}}\left(x_{\varepsilon_{n, m}}\right)\right|>\varepsilon_{n, m}^{-n\left|\alpha_{n}\right|-n}, \forall m \in \mathbb{N}$. 
$\Leftarrow$ : let $K \subset \subset \Omega$ and $\left(x_{\varepsilon}\right)_{\varepsilon} \in K^{(0,1)}$. By assumption, there exist $a, b \in \mathbb{R}$ such that for each $\alpha \in \mathbb{N}^{d}, p_{\alpha, K}\left(u_{\varepsilon}\right) \leq \varepsilon^{-a|\alpha|-b}$, for small $\varepsilon$. Then, for $k:=a+1$ and $p_{n}:=-a n-b$, $\lim _{n} p_{n}+k n=\infty$ and for each $\alpha \in \mathbb{N}^{d},\left|\partial^{\alpha} u_{\varepsilon}\left(x_{\varepsilon}\right)\right| \leq p_{\alpha, K}\left(u_{\varepsilon}\right) \leq \varepsilon^{p_{|\alpha|}}$, for small $\varepsilon$.

\section{$3 \quad \mathcal{G}^{\infty}(\Omega)$ and $\mathcal{G}_{\mathcal{L}_{0}}(\Omega)$}

Our method is based upon a quantitative version of an argument used in [8, Thm. 1.2.3] (cf. also [10, Prop. 1.6] and [17]), which can in fact be traced back to [11].

Proposition 3.1. Let $K \subset \subset \Omega \subseteq \mathbb{R}^{d}$. Suppose that there exists $r \in \mathbb{R}^{+}$such that for each $x \in K$, there exist d line segments of length $r$ containing $x$ in linearly independent directions that are contained in $K$. Let $u \in \mathcal{G}(\Omega)$. If for some $k \in \mathbb{N} \backslash\{0\}, P_{k, K}(u)>$ $P_{k-1, K}(u)$, then $P_{k, K}^{2}(u) \leq P_{k-1, K}(u) P_{k+1, K}(u)$.

Proof. Let first $k=1$. Let $x \in K$. Let $e_{1}, \ldots, e_{d} \in \mathbb{R}^{d}$ be linearly independent unit vectors such that the line segments $\left[x, x+\frac{r}{2} e_{j}\right] \subseteq K$. Denote the directional derivative in the direction $e_{j}$ by $\partial_{e_{j}}$. Let $a \in \mathbb{R}, a>0$. For $\varepsilon \in(0,1)$, by Taylor's formula there exist $\theta_{\varepsilon} \in[0,1]$ such that

$$
\partial_{e_{j}} u_{\varepsilon}(x)=\varepsilon^{-a} u_{\varepsilon}\left(x+\varepsilon^{a} e_{j}\right)-\varepsilon^{-a} u_{\varepsilon}(x)+\frac{\varepsilon^{a}}{2} \partial_{e_{j}}^{2} u_{\varepsilon}\left(x+\varepsilon^{a} \theta_{\varepsilon} e_{j}\right) .
$$

Hence for $\varepsilon \leq \varepsilon_{0}$ (where $\varepsilon_{0}$ does not depend on $x \in K$ ),

$$
\left|\partial_{e_{j}} u_{\varepsilon}(x)\right| \leq 2 \varepsilon^{-a} \sup _{y \in K}\left|u_{\varepsilon}(y)\right|+\varepsilon^{a} \sup _{y \in K}\left|\partial_{e_{j}}^{2} u_{\varepsilon}(y)\right| \leq 2 \varepsilon^{-a} p_{0, K}\left(u_{\varepsilon}\right)+\varepsilon^{a} p_{2, K}\left(u_{\varepsilon}\right) .
$$

Since $e_{1}, \ldots, e_{d}$ are linearly independent, we can write $\partial_{1}, \ldots, \partial_{d}$ as a linear combination (with coefficients independent of $\varepsilon$ and $x$ ) of $\partial_{e_{1}}, \ldots, \partial_{e_{d}}$. Thus there exists $C \in \mathbb{R}$ such that $p_{1, K}\left(u_{\varepsilon}\right) \leq C \varepsilon^{-a} p_{0, K}\left(u_{\varepsilon}\right)+C \varepsilon^{a} p_{2, K}\left(u_{\varepsilon}\right)$, and $P_{1, K}(u) \leq$ $\max \left(e^{a} P_{0, K}(u), e^{-a} P_{2, K}(u)\right)$. Should $P_{2, K}(u) \leq P_{0, K}(u)$, then letting $a \rightarrow 0$ would yield $P_{1, K}(u) \leq P_{0, K}(u)$, contradicting the hypotheses. Hence $P_{2, K}(u)>P_{0, K}(u)$, and we can choose $a>0$ such that $e^{2 a}=P_{2, K}(u) / P_{0, K}(u)$ (since the case $P_{0, K}(u)=0$ is trivial).

If $k \in \mathbb{N} \backslash\{0\}$ arbitrary, the same reasoning can be applied to all $\partial^{\alpha} u$ with $|\alpha|=k-1$ instead of $u$.

Corollary 3.2. (cf. [8, Thm. 1.2.3]) Let $K \subset \subset \Omega \subseteq \mathbb{R}^{d}$. Suppose that there exists $r \in \mathbb{R}^{+}$such that for each $x \in K$, there exist d line segments of length $r$ containing $x$ in linearly independent directions that are contained in $K$. Let $u \in \mathcal{G}(\Omega)$. If for some $k \in \mathbb{N}, P_{k, K}(u)=0$, then $P_{l, K}(u)=0, \forall l \geq k$.

Proof. If $P_{k+1, K}(u) \neq 0$, then $P_{k+1, K}(u)^{2} \leq P_{k, K}(u) P_{k+2, K}(u)=0$ by proposition 3.1, a contradiction. The result follows inductively.

Proposition 3.3. Let $K \subset \subset \Omega$ satisfy the hypothesis of proposition [3.1. Let $u \in$ $\mathcal{G}_{\mathcal{L}_{0}}(K)$. Then $P_{k, K}(u)$ are decreasing in $k$, and

$$
\mathcal{G}^{\infty}(K)=\mathcal{G}_{\mathcal{L}_{0}}(K)=\left\{u \in \mathcal{G}(\Omega): P_{k, K}(u) \leq P_{0, K}(u), \forall k \in \mathbb{N}\right\} .
$$

In particular, $\mathcal{G}^{\infty}(K)$ is closed in $\mathcal{G}(\Omega)$. 
Proof. Let $u \in \mathcal{G}(\Omega)$. If $P_{k, K}(u)$ are not decreasing in $k$, then there exists $k \in \mathbb{N} \backslash\{0\}$ such that $P_{k, K}(u)>P_{k-1, K}(u)>0$ by corollary [3.2. Let $r:=P_{k, K}(u) / P_{k-1, K}(u)>$ 1. By proposition 3.1, $P_{k+1, K}(u) \geq r P_{k, K}(u)$ (in particular, $P_{k+1, K}(u)>P_{k, K}(u)$ ). Inductively, $P_{k+n, K}(u) \geq r^{n} P_{k, K}(u)$, for each $n \in \mathbb{N}$. Thus $\lim \sup _{n \rightarrow \infty} \frac{\ln P_{n+k, K}(u)}{n+k} \geq$ $\lim \sup _{n \rightarrow \infty} \frac{\ln \left(r^{n} P_{k, K}(u)\right)}{n+k}=\ln r>0$, and $u \notin \mathcal{G}_{\mathcal{L}_{0}}(K)$. In particular, $u \notin \mathcal{G}^{\infty}(K)$.

The fact that $\mathcal{G}^{\infty}(K)$ is closed follows by continuity of $P_{k, K}$.

Theorem 3.4. $\mathcal{G}^{\infty}(\Omega)=\mathcal{G}_{\mathcal{L}_{0}}(\Omega)$ is closed in $\mathcal{G}(\Omega)$. In particular, $\mathcal{G}^{\infty}(\Omega)$ is not dense in $\mathcal{G}(\Omega)$.

Proof. $\mathcal{G}^{\infty}(\Omega)=\bigcap_{K} \mathcal{G}^{\infty}(K)$, where $K$ runs over all compact subsets of $\Omega$ that are a finite union of $d$-dimensional cubes parallel with the coordinate axes (hence satisfying the hypothesis of proposition [3.1), and similarly for $\mathcal{G}_{\mathcal{L}_{0}}(\Omega)$. The conclusions follow from proposition 3.3 .

$4 \quad \mathcal{G}_{\mathcal{L}_{a}}(\Omega), a>0$

Proposition 4.1. Let $K \subset \subset \Omega$ satisfy the hypothesis of proposition [3.1. Let $a \in \mathbb{R}$, $a \geq 1$. Then

$$
\begin{aligned}
\left\{u \in \mathcal{G}(\Omega):(\exists c \in \mathbb{R})\left(P_{k, K}(u) \leq c a^{k},\right.\right. & \forall k \in \mathbb{N})\} \\
& =\left\{u \in \mathcal{G}(\Omega): P_{k+1, K}(u) \leq a P_{k, K}(u), \forall k \in \mathbb{N}\right\}
\end{aligned}
$$

In particular, this describes a closed subset of $\mathcal{G}(\Omega)$.

Proof. Let $u \in \mathcal{G}(\Omega)$. If $P_{k+1, K}(u)>a P_{k, K}(u)$, for some $k \in \mathbb{N}$, then $P_{k, K}(u)>0$ by corollary 3.2. Let $r:=P_{k+1, K}(u) / P_{k, K}(u)>a$. By proposition 3.1, $P_{k+n, K}(u) \geq$ $r^{n} P_{k, K}(u)$, for each $n \in \mathbb{N}$. Thus $\lim \sup _{n \in \mathbb{N}} P_{n, K}(u) / a^{n} \geq \lim \sup _{n \in \mathbb{N}} \frac{r^{n-k} P_{k, K}(u)}{a^{n}}=$ $+\infty$.

The other inclusion is clear.

Theorem 4.2. Let $a \in \mathbb{R}, a>0$. Then $\mathcal{G}_{\mathcal{L}_{a}}(\Omega)$ is not dense in $\mathcal{G}(\Omega)$.

Proof. $\mathcal{G}_{\mathcal{L}_{a}}(\Omega) \subseteq \bigcap_{K}\left\{u \in \mathcal{G}(\Omega):(\exists c \in \mathbb{R})\left(P_{k, K}(u) \leq c e^{a k}, \forall k \in \mathbb{N}\right)\right\}=: \mathcal{A}$, where $K$ runs over all compact subsets of $\Omega$ that are a finite union of $d$-dimensional cubes parallel with the coordinate axes. The set $\mathcal{A}$ is closed by proposition 4.1 and is a strict subset of $\mathcal{G}(\Omega)$.

\section{$5 \quad$ Sublinear generalized functions}

In order to investigate the density of the algebra of sublinear generalized functions, we start with the following proposition (see also [16, Prop. 4.3.1]):

Proposition 5.1. Let $\psi \in \mathcal{C}^{\infty}\left(\mathbb{R}^{d}\right)$ with $\psi(x)=0$ if $|x| \geq 1$ and $\int_{\mathbb{R}^{d}} \psi=1$. Denote by $\psi_{\varepsilon}(x):=\varepsilon^{-d} \psi(x / \varepsilon)$, for each $\varepsilon \in(0,1)$. If $u=\left[\left(u_{\varepsilon}\right)_{\varepsilon}\right] \in \mathcal{G}(\Omega)$, then $\lim _{n \rightarrow \infty}\left[\left(u_{\varepsilon} \star\right.\right.$ $\left.\left.\psi_{\varepsilon^{n}}\right)_{\varepsilon}\right]=u$. 
Proof. Let $n \in \mathbb{N}$ and $K \subset \subset \Omega$. Then $u_{\varepsilon} \star \psi_{\varepsilon^{n}}(x)=\int_{|t| \leq \varepsilon^{n}} u_{\varepsilon}(x-t) \psi_{\varepsilon^{n}}(t) d t$ is well-defined as soon as $d\left(x, \mathbb{R}^{d} \backslash \Omega\right)>\varepsilon^{n}$. For small $\varepsilon, d(K, \mathbb{R} \backslash \Omega)>\varepsilon^{n}$ and thus $\left(u_{\varepsilon} \star \psi_{\varepsilon^{n}}\right)_{\mid K}$ can be extended to a $\mathcal{C}^{\infty}(\Omega)$-function. Independent of the extension, by the mean value theorem,

$$
\begin{aligned}
p_{k, K}\left(u_{\varepsilon} \star \psi_{\varepsilon^{n}}-u_{\varepsilon}\right)=\sup _{x \in K,|\alpha|=k}\left|\left(\partial^{\alpha} u_{\varepsilon}\right) \star \psi_{\varepsilon^{n}}(x)-\partial^{\alpha} u_{\varepsilon}(x)\right| \\
=\sup _{x \in K,|\alpha|=k}\left|\int_{|t| \leq \varepsilon^{n}}\left(\partial^{\alpha} u_{\varepsilon}(x-t)-\partial^{\alpha} u_{\varepsilon}(x)\right) \psi_{\varepsilon^{n}}(t) d t\right| \leq \varepsilon^{n} p_{k+1, K+r}\left(u_{\varepsilon}\right) \int_{\mathbb{R}^{d}}|\psi|
\end{aligned}
$$

for small $\varepsilon$, where $r>0(r \in \mathbb{R})$ such that $K+r=\left\{x \in \mathbb{R}^{d}: d(x, K) \leq r\right\} \subset \subset \Omega$.

Proposition 5.2. Let $\mathcal{A}$ be the set of all $u=\left[\left(u_{\varepsilon}\right)_{\varepsilon}\right] \in \mathcal{G}_{c}(\Omega)$ for which

$$
(\exists N \in \mathbb{N})(\forall K \subset \subset \Omega)(\forall k \in \mathbb{N})\left(p_{k, K}\left(u_{\varepsilon}\right) \leq \varepsilon^{-N k-N}, \text { for small } \varepsilon\right) .
$$

Then $\mathcal{A}$ is dense in $\mathcal{G}(\Omega)$.

Proof. Let $u \in \mathcal{G}_{c}(\Omega)$. Then there exists a representative $\left(u_{\varepsilon}\right)_{\varepsilon}$ of $u$ and $L \subset \subset \Omega$ such that $\operatorname{supp} u_{\varepsilon} \subseteq L$, for each $\varepsilon$. For each $K \subset \subset \Omega$ and $k \in \mathbb{N}$,

$$
\begin{aligned}
p_{k, K}\left(u_{\varepsilon} \star \psi_{\varepsilon^{n}}\right)=\sup _{x \in K,|\alpha|=k} \mid u_{\varepsilon} & \star \partial^{\alpha}\left(\psi_{\varepsilon^{n}}\right) \mid \\
& \leq \varepsilon^{-n k} \sup _{x \in L}\left|u_{\varepsilon}(x)\right| \max _{|\alpha|=k} \int_{\mathbb{R}^{d}}\left|\partial^{\alpha} \psi\right| \leq \varepsilon^{-n k-1} \sup _{x \in L}\left|u_{\varepsilon}(x)\right|,
\end{aligned}
$$

for small $\varepsilon$. Thus $\left[\left(u_{\varepsilon} \star \psi_{\varepsilon^{n}}\right)_{\varepsilon}\right] \in \mathcal{A}$. By proposition $\left[5.1, \mathcal{A}\right.$ is dense in $\mathcal{G}_{c}(\Omega)$. Further, $\mathcal{G}_{c}(\Omega)$ is dense in $\mathcal{G}(\Omega)$ (for $u \in \mathcal{G}(\Omega), u=\lim _{n \rightarrow \infty} u \chi_{n}$, where $\chi_{n} \in \mathcal{D}(\Omega)$ with $\chi_{n}(x)=1, \forall x \in K_{n}$, where $\left(K_{n}\right)_{n \in \mathbb{N}}$ is a compact exhaustion of $\Omega$ ).

Theorem 5.3. The subalgebra of sublinear generalized functions is dense in $\mathcal{G}(\Omega)$.

Proof. With the notations of proposition 5.2, $\mathcal{A} \subseteq\{u \in \mathcal{G}(\Omega): u$ is sublinear $\}$.

Acknowledgment. We are grateful to D. Scarpalézos for very useful discussions.

\section{References}

[1] J. Aragona, R. Fernandez, S. O. Juriaans, A discontinuous Colombeau differential calculus, Monatsh. Math. 144, 1329 (2005).

[2] H. Biagioni, A Nonlinear Theory of Generalized Functions, Lec. Notes Math. 1421, Springer, 1990.

[3] A. Delcroix, Generalized integral operators and Schwartz kernel type theorems, J. Math. Anal. Appl. 306(2), 481-501 (2005).

[4] A. Delcroix, Regular rapidly decreasing nonlinear generalized functions. Application to microlocal regularity, J. Math. Anal. Appl. 327(1), 564-584 (2007). 
[5] N. Dapić, S. Pilipović and D. Scarpalézos, Microlocal analysis of Colombeau's generalized functions: propagation of singularities, J. Analyse Math. 75, 51-66 (1998).

[6] C. Garetto, Topological structures in Colombeau algebras: topological $\widetilde{\mathbb{C}}$-modules and duality theory, Acta Appl. Math. 88(1), 81-123 (2005).

[7] C. Garetto, G. Hörmann, Microlocal analysis of generalized functions: pseudodifferential techniques and propagation of singularities, Proc. Edin. Math. Soc. 48, 603-629 (2005).

[8] M. Grosser, M. Kunzinger, M. Oberguggenberger, R. Steinbauer, Geometric theory of generalized functions with applications to general relativity, Kluwer, 2001.

[9] G. Hörmann, M. Kunzinger, Microlocal properties of basic operations in Colombeau algebras, J. Math. Anal. Appl. 261, 254-270 (2001).

[10] G. Hörmann, M. Oberguggenberger, S. Pilipović, Microlocal hypoellipticity of linear partial differential operators with generalized functions as coefficients, Trans. Amer. Math. Soc. 358, 3363-3383 (2006).

[11] E. Landau, Einige Ungleichungen für zweimal differentiierbare Funktionen, Proc. London Math. Soc. Ser. 2, 13, 43-49 (1913-1914).

[12] M. Nedeljkov, S. Pilipović, D. Scarpalézos, The linear theory of Colombeau generalized functions, Pitman Res. Not. Math. 385, Longman Sci. Techn., 1998.

[13] M. Oberguggenberger, Multiplication of distributions and applications to partial differential equations, Pitman Res. Not. Math. 259, Longman Sci. Techn., 1992.

[14] M. Oberguggenberger, Regularity theory in Colombeau algebras, Bull. Acad. Serbe Sci. Arts (Cl. Sci. Math. Natur.) T. CXXXIII 31, 147-162 (2006).

[15] S. Pilipović, D. Scarpalézos, V. Valmorin, Real analytic generalized functions, Monatsh. Math. 156(1), 85-102 (2009).

[16] D. Scarpalézos, Topologies dans les espaces de nouvelles fonctions généralisées de Colombeau. $\widetilde{\mathbb{C}}$-modules topologiques, Université Paris 7, 1992.

[17] H. Vernaeve, Pointwise characterizations in generalized function algebras, Monatsh. Math. 158(2), 195-213 (2009). 